\title{
Comunicación, Salud y Tecnología: mHealth
}

\section{Palabras clave}

Comunicación

salud

tecnología

aplicaciones

apps

teléfono móvil

smartphone
José Ignacio Niño González

Universidad Complutense de Madrid

\author{
Benito Fernández Morales \\ Universidad de Valencia
}

Autor para correspondencia: José Ignacio Niño González. Universidad Complutense de Madrid, Facultad de

Ciencias de la Información (Departamento de Comunicación Audiovisual II). Avda Complutense s/n.

Correo electrónico: josenino@ucm.es 


\section{Communication, Health and Technology: the road to mHealth}

\begin{tabular}{c}
\hline Keywords \\
\hline \\
Communication health \\
technology \\
applications \\
apps \\
mobile phone \\
smartphone
\end{tabular}

\begin{abstract}
The technological development experienced in recent decades has led to a profound change in a society currently consists of individuals with a high degree of dependence on devices that change their behaviors and improve their daily quality of life. With a smartphone in hand not only have a tool to communicate, we have in our possession a tool through mobile or more commonly known as apps identified applications gives us access to a large universe of resources. The contribution of mobile applications to health occurs in different policy areas and in this paper we focus on the fundamentals that define the capabilities of mHealth.
\end{abstract}

Cómo citar el artículo

Niño González, J. I. y Fernández Morales, B. (2015). Comunicación, Salud y Tecnología: mHealth. Revista de Comunicación y Salud, Vol. 5, pp. 149-158.

DOI: http://doi.org/10.35669/revistadecomunicacionysalud.2015.5.149-158 


\section{Introducción}

A lo largo de la historia los sistemas de comunicación han ido evolucionando en función de las necesidades y las tecnologías existentes. La construcción de las grandes vías romanas, el telégrafo, el teléfono y en la actualidad las telecomunicaciones y la informática muestran como el ser humano ha sido capaz de progresar en busca de soluciones eficaces.

Al igual que ha sucedido con la comunicación, las ciencias de la salud han experimentado un profundo proceso de transformación desde los primeros curanderos de las tribus hasta llegar a los actuales grandes centros médicos dotados de las más vanguardistas tecnologías. Cuanto mayor es el desarrollo de una sociedad, mayor es el grado de evolución de su medicina, y el estetoscopio, los rayos $\mathrm{X}$, las vacunas, la penicilina, la medicina nuclear, las resonancias magnéticas y los protocolos de actuación médica son ejemplos representativos del avance de la medicina que, junto con los medicamentos personalizados, la implantación de electrodos y micro chips, conforman el panorama de la tecnología médica de hoy en día.

En la evolución de nuestra sociedad, la presencia del médico y los medicamentos constituyen una necesidad de primer orden asumida y demandada por todos. La prontitud en la intervención, el acierto en el diagnóstico y la prevención, son las respuestas que las ciencias de la salud han dado siempre a esas demandas sociales, y para lograrlo se han apoyado en la comunicación y en las innovaciones tecnológicas. Así la mejora de las carreteras y las ambulancias ayudan en la prontitud en la intervención médica, la radiología, los TAC y escáneres en el diagnóstico de enfermedades, y los softwares e internet en la mejora de los protocolos de actuación mediante un intercambio rápido de información y conocimiento que es un pilar fundamental en la lucha contra las enfermedades. En el ámbito de la prevención es donde más evidentemente la tecnología, la comunicación y las ciencias de la salud se dan la mano, especialmente en la capacidad y rapidez de divulgación que aporta internet: la posibilidad de comunicar la imagen y el sonido en tiempo real con un simple smartphone o la situación en espacios geográficos de toda la información endémica de continentes enteros, mediante los sistemas G.I.S ( Geographis Informations System) que permiten almacenar todo tipo de información médica de países en bases de datos, proyectándola sobre un mapa geográfico, e interrelacionarla con otras, cruzando datos que permitan intervenciones y tomas de decisiones rápidas y eficientes.

Los sistemas de comunicación es la manera que hemos inventado los humanos para salvar las distancias que nos separaban de las personas, los recursos y los conocimientos. Actualmente podemos decir "que ya no hay distancias", ya que los novedosos sistemas introducidos en la práctica médica permiten actuar de forma rápida incluso en situaciones en las que se requiere una intervención en remoto soportada por las nuevas tecnologías. La mejora continua en los procesos de diagnóstico y los protocolos médicos, gracias a los intercambios de datos y conocimientos entre los distintos centros médicos y profesionales les hacen más eficientes, sin olvidar la personalización de los tratamientos que mejoran la calidad de vida de los pacientes.

$Y$ si bien es cierto que ya no hay distancias y que todo avanza muy rápidamente, en realidad de lo que hoy carecemos es de una variable difícil de gestionar, el tiempo. Es precisamente en este campo donde las nuevas tecnologías y los sistemas de comunicación pueden ayudarnos y en concreto 
con la Telemedicina abordaremos un campo que representa uno de los pasos más importantes para alcanzar el objetivo de acercar las soluciones médicas a todo el mundo mediante su capacidad para llegar a sitios que son inaccesibles por su ubicación geográfica con un tiempo de respuesta casi inmediato. Además permite transmitir, metodologías y conocimientos científicos de actuación médica para ser aplicados en pacientes que de otro modo nunca podrían tener acceso a estos tratamientos.

La Telemedicina ha sido y es el gran impulsor de la universalización de la práctica de la medicina, pues no tiene aranceles de entrada, no hay censuras previas y focaliza como primer nivel de importancia en la salud del enfermo. Por tanto creemos que es uno de los elementos esenciales en la democratización del cuidado de la salud, es una puerta abierta a la gestión del conocimiento que permite acceder a todo tipo de datos, técnicas y profesionales casi de manera instantánea, monitorizar a los pacientes y hacer un seguimiento en tiempo real de sus constantes médicas.

El desarrollo tecnológico es el germen de la creación de unas comunidades virtuales que se han convertido en un aliado fundamental de la medicina preventiva, pues permiten llegar a millones de ciudadanos en tiempo real para movilizarlos con un fin concreto e interrelacionar a los distintos profesionales de la comunidad científica para unificar criterios y opiniones. De este modo, la comunidad virtual tiene acceso a un valioso volumen de conocimientos y opiniones relacionados con las ciencias de la salud, pero también es la entrada para aquellas comunidades de opinión que pueden ser peligrosas para los ciudadanos y para las políticas generales sobre salud por la influencia que tienen sobre colectivos muy amplios. Esto supone que al igual que se pueden crear corrientes de opinión favorables a los planes generales de prevención de enfermedades, también pueden ser el acceso para todo tipo de manipulación de la población por parte de intereses poco claros que implicarían un enorme perjuicio como pueden ser aspectos como la auto medicación.

Como podemos ver, la comunicación es imprescindible en nuestra sociedad y esencial para las ciencias de la salud y la medicina en particular. Pero una comunicación no verificada puede traer consigo consecuencias no deseadas muy importantes, por lo que debe ser tratada de una manera profesional y responsable. Hay que asegurarse que el mensaje y su contenido es entendido en sus justos términos o si existe alguna posibilidad de que pueda ser tergiversado y actuar en consecuencia.

Con la evolución de las tecnologías se está produciendo una verdadera revolución en la medicina con la irrupción de dispositivos dotados de un enorme potencial comunicativo. En este sentido, el teléfono móvil y el entorno de las aplicaciones móviles ofrecen un inmenso abanico de posibilidades para el mundo de la salud que se concreta en las llamadas aplicaciones mHealth.

\section{El teléfono móvil}

El desarrollo tecnológico experimentado en las últimas décadas ha provocado un drástico cambio en una sociedad actualmente formada por individuos con un alto grado de dependencia hacia dispositivos que modifican sus comportamientos y que mejoran día a día su calidad de vida. El poderoso vínculo creado entre individuo y dispositivo no es gratuito, se sustenta en una estrecha relación que aporta un alto grado de "satisfacción" al usuario que ve 
en su "máquina" un aliado que le facilita sus tareas cotidianas. Como establecen Davis, F. \& Venkatesh, V. (1989) en su "Teoría de la Aceptación de la Tecnología" los seres humanos son proclives a aceptar cualquier nueva tecnología siempre y cuando se perciba con nitidez que este nuevo reto contribuye a facilitarles la vida. El exponente más claro de la anterior teoría es el teléfono móvil, un dispositivo con una presencia tan amplia que confirma que ya prácticamente nadie puede prescindir de él. Según el Instituto Nacional de Estadística (INE), a 1 de enero de 2015 había censadas en España 46.439.864 de personas, dato que si lo relacionamos con el ofrecido por la Comisión Nacional del Mercado de las Comunicaciones (CNMC) que en abril del 2015 cifraba en 50,37 millones las líneas de móviles dadas de alta nos permite darnos cuenta de la verdadera dimensión que tiene este dispositivo en la realidad social actual. Nunca en la historia de la humanidad, una tecnología había tenido el nivel de penetración y adopción que ha experimentado el teléfono móvil y sin lugar a dudas esta circunstancia esta intrínsecamente ligada con una de las cinco variables identificadas por Schiffman y Kanuk (1997) que parece pueden influir en el grado de aceptación de nuevos productos, la observabilidad, explicada como la facilidad con la que se perciben los beneficios. Evidentemente todos entendemos en mayor o menor medida que el dispositivo móvil nos ayuda, y en consecuencia nuestro pensamiento pragmático nos lleva ineludiblemente a su adopción.

\section{Las aplicaciones móviles}

Con un smartphone en la mano no solo tenemos un dispositivo para llamar en cuanto lo necesitemos, tenemos en nuestro poder una herramienta que, gracias a las conocidas aplicaciones móviles o más comúnmente identificadas como apps, nos permite acceder a un gran universo de recursos. ¿Pero cómo podríamos definir estas apps de un modo sencillo? Una app móvil es un software diseñado de forma específica para ser instalado en dispositivos móviles o tablets y que nos ofrece una solución o función concreta. El objetivo de una app no es otro que facilitarnos la consecución de una tarea determinada o ayudarnos en determinadas gestiones. Existen más de un millón de aplicaciones de muy diferente tipología que dan respuestas en muchos ámbitos: apps de noticias, de juegos, de mensajería instantánea, de tiendas, etc... en definitiva un amplio abanico de opciones en el que es fácil encontrar la aplicación que se busca para cubrir una necesidad concreta. De acuerdo con el "50 Informe Estado de las Apps en España" elaborado en 2014 por The App Date, hay 23 millones activos de usuarios de apps, se descargan 3,8 millones de apps al día, hay una media de 39 apps instaladas por smartphone y la aplicación LogosQuiz ha alcanzado las 34.059.076 descargas. Unos datos contundentes que expresan el gran interés que existe por unas aplicaciones que se consolidan en un sector que muestra un crecimiento exponencial principalmente en apps centradas en el mundo del ocio y el consumo, pero que consciente de las grandes oportunidades que pueden desarrollarse en otras vertientes se abre camino hacia nuevas áreas de explotación. En este sentido, y dada la importancia que tiene para el ser humano, el mundo de la salud no permanece ajeno a estas nuevas tendencias tecnológicas y en consecuencia se han ido desarrollando conceptos como el de mHealth. 


\section{Las aplicaciones mHealth}

Entendemos la mHealth como aquella parte de la eHealth (salud electrónica/esalud) y la OMS la define como:

mHealth es la práctica de la medicina y la salud pública soportada por dispositivos móviles como teléfonos móviles, dispositivos de monitorización de pacientes, asistentes personales digitales y otros dispositivos inalámbricos.

Y como señala el director del Observatorio Zeltia, Dr. Fernando Mugarza:

Dentro de este ámbito de las nuevas tecnologías asociadas a la salud, el fenómeno de las apps es sin duda el que está haciendo que tanto el paciente como el profesional adquieran otra dimensión. Compartir el conocimiento y hacerlo además de una forma ilustrativa, fácilmente comprensible y a través de una herramienta tecnológica absolutamente versátil, de fácil manejo y usabilidad, es algo que conforma un espacio de comunicación abierto, que permite ya no sólo entender, sino, además, compartir experiencias en el entorno que más preocupa al ser humano, el de su propia salud.

La aportación de las aplicaciones móviles a la sanidad se produce en diferentes ámbitos de actuación, pero en este trabajo nos centraremos en dos aspectos que consideramos fundamentales ya que en nuestra opinión son los que marcan de un modo definitorio las capacidades de la mHealth. En primer lugar debemos hablar del concepto de "empoderamiento", término con el que se da contenido al nuevo papel que deben asumir los individuos en su relación con su salud. El médico no debe entenderse como el único "guardián" de la misma, es el individuo el que debe ser consciente de que tiene que asumir un rol activo y comenzar a preocuparse por salud adquiriendo unos hábitos de autovigilancia y control. En este sentido, las aplicaciones móviles pueden cumplir una misión básica y dotar a los ciudadanos de unas herramientas de control, cuidado y prevención que les permitan participar activamente en el proceso. En segundo lugar, y aunque ya lo hemos enunciado anteriormente, desarrollar el aspecto preventivo y decir que si la UE abordase rigurosas políticas en el campo de la $m H$ ealth los costes sanitarios se podrían reducir en una cifra cercana a los 100.000 millones de euros. Como ejemplos de esta realidad, indicar los datos recogidos en el último estudio sobre la "Sociedad de la Información en España" de la Fundación Telefónica en el que se indica que la utilización de tecnologías móviles reduciría el coste sanitario per cápita en un 18\% y hasta un $35 \%$ si se habla del tratamiento de pacientes crónicos en el año 2017. 
El número de apps en el entorno de la salud en muy considerable. El último estudio del IMS Institute for Healthcare Informatics, fija en 97.000 las aplicaciones existentes a nivel mundial de las que un $70 \%$ se dirigen al público en general en los segmentos de bienestar y ejercicio físico mientras que el 30\% restante se dirige al sector de los profesionales sanitarios y pacientes. Por funcionalidades las más comunes son las apps desarrolladas que aportan información (39,8\%), seguidas por las que dan instrucciones de uso $(21,4 \%)$ y registran datos del usuario (18,7\%). Si nos fijamos únicamente en las aplicaciones para la salud desarrolladas en español, el primer "Informe de las 50 mejores apps de salud en español", elaborado por The App Date, con el impulso del Observatorio Zeltia y la colaboración de la Cátedra conjunta ZeltiaUniversidad Rey Juan Carlos de Madrid, Wake App Health y Red de Innovación (RDi) muestra que el $24 \%$ son informativas, el $22 \%$ monitorizan parámetros físicos, el 18\% facilitan el seguimiento del tratamiento, el $16 \%$ relacionadas con la educación, $14 \%$ con la gestión y $6 \%$ con ayuda al diagnóstico.

Respecto al amplio repertorio de aplicaciones existentes hay que señalar como dato importante que el $66 \%$ de las aplicaciones en español son gratuitas, pero hay un 34\% de ellas que son de pago. La dimensión económica de estas apps es muy relevante ya que se estima que durante 2015 un tercio de los usuarios de smartphones tendrán instaladas como mínimo una de estas aplicaciones y estos datos de uso arrojan una proyección de negocio en España de 4.000 millones de euros y lo que es más relevante, en un sector con una tendencia de crecimiento en los próximos años realmente muy importante.

\section{Las $\mathbf{1 0}$ mejores aplicaciones de salud}

No estaría completo este trabajo si no hiciésemos un breve repaso a las calificadas como mejores 10 apps de salud según los datos obtenidos en el anteriormente mencionado "Informe de las 50 mejores apps de salud en español obstante, y que por orden alfabético son: 30 años de VIH, Abah, Contigo', Dermomap, Doctoralia', Endomondo, iDoctus, Sanitas Embarazo, Social Diabetes y Universal Doctor Speaker.

1/ 30 años de VIH: guía para obtener información sobre la enfermedad y los avances médicos en su tratamiento en los últimos treinta años.

2/ Ablah: Dirigida a pacientes con autismo y dificultades en el habla y dirigida a mejorar sus capacidades comunicativas. Premiada en los Vodafone Smart Accesibility Awards 2012 como mejor aplicación de Bienestar y mejor app española 2010 por The App Date.

3/ Contigo: Centrada en 16 mujeres que han superado un cáncer de mama y ayudan con su experiencia a otras mujeres que padecen la enfermedad. Obtuvo en 2013 el premio a la mejor app de salud en los Vodafone Mobile for Good Europe Awards. 
4/ Dermomap: Dirigida a profesionales, ayuda a diagnosticar enfermedades de la piel. En 2013 consiguió el Premio Ideas Sanitas a la mejor aplicación móvil de salud.

5/ Doctoralia: App que permite tanto a profesionales como a pacientes gestionar citas médicas, encontrar especialistas en función del seguro médico que se tiene y opinar sobre los médicos. Premiada en el App Circus de 2012.

6/ Endomondo: Aplicación de carácter social en la que el usuario registrándose a través de Facebook accede a un entrenador personal y puede compartir con el resto de usuarios ejercicios, rutas, etc. Incorpora GPS y medición del ritmo cardíaco.

7/ iDoctus: Ayuda a la práctica clínica del médico e incluye servicios de documentación, bases de datos de medicamentos actualizadas, archivo multimedia y apoyo al diagnóstico. Ganadora en SeedRocket 2011 y finalista en Ideas Sanitas 2013.

8/ Sanitas Embarazo: Ofrece información sobre las etapas del embarazo con modelos interactivos en 3D, consejos elaborados por especialistas y permite planificar las visitas al ginecólogo.

9/ Social Diabetes: Herramienta de control de la diabetes tipo 1 y 2 que permite a los pacientes calcular las dosis de hidratos y la administración de insulina y a los médicos monitorizar al paciente en remoto.

10/ Universal Doctor Speaker: Cuando un paciente viaja a otro país para una consulta, este traductor médico apoyado en ilustraciones y locuciones facilita la comunicación.

\section{Conclusiones}

Después de revisar el entorno de la mHealth no quedan muchas dudas sobre su potencial materializado en un intenso presente y con una proyección de futuro realmente optimista. Pero como en cualquier otro ámbito de la vida, no todo son luces en este sector, existen algunos puntos que arrojan sombras y que la mHealth debe tener muy en cuenta solucionar si quiere llegar a explotar todo su potencial. En concreto hablamos de tres puntos:

1/ Integración en el sistema sanitario: es necesario un esfuerzo conjunto de todos los actores que intervienen en el ámbito de la mHealth para generar políticas que conlleven de un modo estructurado la adopción como práctica regulada de estas aplicaciones en el sistema sanitario. Difícilmente estas herramientas tendrán un futuro solvente si no hay una concienciación integradora, y sin duda desde el ámbito gubernamental tiene que existir un apoyo claro y firme. 
2/ Impulso al desarrollo y visibilidad: Javier Navarro CEO de The App Date España, comenta que más del $50 \%$ de las aplicaciones disponibles tuvieron menos de 500 descargas y que sólo cinco registraron el $15 \%$ de todas las descargas obtenidas en la categoría de salud. Aunque esta situación responde en gran medida a la misma realidad que sufre el conjunto global de aplicaciones móviles en todas las categorías, seria esencial por su valor social crear programas destinadas a impulsar el desarrollo y a dar visibilidad a este tipo de aplicaciones con objeto de que los potenciales usuarios las conozcan y puedan interaccionar con ellas y que los desarrolladores tengan un soporte para realizar su trabajo. Para ello es necesario un apoyo institucional, especialmente necesario en aquellas aplicaciones de carácter gratuito que aporten valor y que no solo necesiten darse a conocer, sino que además puedan recibir un rendimiento económico por su desarrollo. De este modo, muchos desarrolladores con poca capacidad económica pero con grandes ideas podrían lanzarse a la creación de aplicaciones y muchos usuarios podrían mejorar su salud. No olvidemos que al reivindicar un apoyo en programas institucionales no podemos dejar de pensar en una cifra ya reflejada: las tecnologías móviles reduciría el coste sanitario per cápita en un $18 \%$. No parece desacertado pensar que una inversión en este tipo de actividades sería muy rentable a corto plazo.

3/ Sello de calidad: y por último e intrínsecamente relacionado con los dos puntos anteriores, es necesario establecer los mecanismos reguladores que permitan certificar que las aplicaciones móviles en el campo de la salud son seguras para su uso por parte de los ciudadanos y que cumplen los estándares de calidad que se fijen para validarlas. Mencionar en esta línea la iniciativa de la Agencia de Calidad Sanitaria de Andalucía, quien con su distintivo App Saludable es una referencia que sin duda puede marcar el camino a seguir. 


\section{Referencias}

Big data: The next frontier for innovation, competition, and productivity. McKinsey Global Institute. Junio 2011.

Davis, F. \& Venkatesh, V. (1989). User acceptance of computer technology: a comparision of two theoretical models. Management Science Magazine. Vol. 35, pp. 982-1003.

IAB Spain (International Advertising Bureau) (2013). Guía de la Eficacia Mobile.

Informe de las 50 mejores apps de salud en español, elaborado por The App Date, con el impulso del Observatorio Zeltia y la colaboración de la Cátedra conjunta Zeltia-Universidad Rey Juan Carlos de Madrid, Wake App Health y Red de Innovación (RDi). Enero 2014.

Informe sobre las apps en España 2013. The App Date. Septiembre 2013.

mHealth in an mWorld: How mobile technology is transforming health care, Deloitte Center for Health Solutions. 2012.

Patient Apps for Improved Healthcare. From Novelty to Mainstream. IMS Institute for Healthcare Informatics. Octubre 2013.

Patient Monitoring Devices Market to 2017- Increasing Use of Wireless Remote Patient Monitoring to be the Key Technology Trend. GBI Research. Marzo 2012.

Schiffman, L. G. \& Kanuk, L. L. (1997). Comportamiento del consumidor. México: Prentice Hall.

Sociedad de la Información en España 2013. Fundación Telefónica. Enero 2014.

Topol, E. (2015). The Patient Will See You Now: The Future of Medicine is in Your Hands. Basic Books.

MacManus, R. (2014). Trackers: How technology is helping us monitor and improve our Health. Rowman \& Littlefield Publishers. 